\title{
AN ALMOST POISSON STRUCTURE FOR THE GENERALIZED RIGID BODY EQUATIONS
}

\author{
Anthony M. Bloch ${ }^{*, 1}$ Peter E. Crouch ${ }^{* *, 2}$ \\ Jerrold E. Marsden ${ }^{* * * 3}$ Tudor S. Ratiu ${ }^{* * * *, 4}$ \\ * Department of Mathematics, University of Michigan, \\ Ann Arbor, MI 48109 \\ ** Center for Systems Science and Engineering, Arizona State \\ University, Tempe, AZ 85287 \\ *** Control and Dynamical Systems, California Institute of \\ Technology 107-81, Pasadena, CA 91125 \\ **** Départément de Mathématiques, École polytechnique fédérale \\ de Lausanne, CH - 1015 Lausanne, Switzerland
}

\begin{abstract}
In this paper we introduce almost Poisson structures on Lie groups which generalize Poisson structures based on the use of the classical Yang-Baxter identity. Almost Poisson structures fail to be Poisson structures in the sense that they do not satisfy the Jacobi identity. In the case of cross products of Lie groups, we show that an almost Poisson structure can be used to derive a system which is intimately related to a fundamental Hamiltonian integrable system - the generalized rigid body equations. Copyright $(\mathrm{C}) 2000$ IFAC
\end{abstract}

Keywords: Almost Poisson structure, Poisson Lie groups, Sklyanin bracket, reduction, the rigid body, integrable system.

\section{INTRODUCTION}

The theory of Poisson-Lie groups is of great interest in the theory of both classical and quantum systems. The basic theory of Poisson manifolds is discussed, for example, in Weinstein [1983], while the theory of Poisson-Lie groups (groups endowed with a Poisson structure) was developed in the work of Drinfeld [1983], Semenov-Tian-Shansky [1985], and Lu and Weinstein [1990], among other works.

\footnotetext{
1 Research partially supported by NSF grant DMS9803181, AFOSR grant F49620-96-1-0100, and an NSF group infrastructure grant at the University of Michigan 2 Work supported in part by NSF grant DMS 91011964 and NATO grant CRG 910926

3 Research partially supported by the California Institute of Technology and NSF grant DMS-9802106

4 Work supported in part by NSF grant DMS-98-02378 and the Swiss NSF
}

A key aspect of much of this work is the socalled modified Yang Baxter equation. Operators satisfying this equation are classical $r$-matrices and may be used to define Poisson structures on Lie groups. This yields, for example, the Sklyanin bracket which is used in Deift and Li [1991] to analyze the Toda lattice flow and its relationship to the SVD (singular value decomposition) flow.

To our knowledge no such Poisson-Lie structure has been found for other key classical integrable systems such as the generalized rigid body equations. In this paper we define a structure on a group which is not Poisson-Lie, but is very close to being Poisson in a way which we will make precise and which yields the generalized rigid body equations in a new form. This structure is based on a form of the equations originally derived in the work of Bloch and Crouch [1996] and Bloch, Brockett, and Crouch [1997] and inspired by op- 
timal control problems (see also Brockett [1994]). A connection of this work to discrete rigid body equations may be found in Bloch, Crouch, Marsden, and Ratiu [1998], [2000].

\section{PRELIMINARY DEFINITIONS AND BACKGROUND THEORY}

In this section we introduce some notation and review some principal results on Poisson structures. Let $G$ be a Lie group with Lie algebra $\mathfrak{g}$, and let $\langle$,$\rangle be an A d$-invariant inner product on $\mathfrak{g}$. A key object in defining Poisson structures on groups is the notion of left and right derivatives of $C^{\infty}$ functions on $G$ (see Semenov-Tian-Shansky [1985] and Deift and Li [1991]).

Definition 1. Let $\phi \in C^{\infty}(G)$. Then the right derivative $D_{R} \phi_{g}^{\#}$ of $\phi$ at $g$ is given by

$$
D_{R} \phi_{g}(X)=\left.\frac{d}{d t}\right|_{t=0} \phi\left(g e^{t X}\right)=\left\langle D_{R} \phi_{g}^{\#}, X\right\rangle
$$

where $X \in \mathfrak{g}, D_{R} \phi_{g} \in \mathfrak{g}^{*}$ and $D_{R} \phi_{g}^{\#} \in \mathfrak{g}$. Similarly, the left derivative of $\phi \in C^{\infty}(G)$ is given by

$$
D_{L} \phi_{g}(X)=\left.\frac{d}{d t}\right|_{t=0} \phi\left(e^{t X} g\right)=\left\langle D_{L} \phi_{g}^{\#}, X\right\rangle
$$

where $X \in \mathfrak{g}, D_{L} \phi_{g} \in \mathfrak{g}^{*}$ and $D_{L} \phi_{g}^{\#} \in \mathfrak{g}$.

Let $A d_{g}: \mathfrak{g} \rightarrow \mathfrak{g}, A d_{g}(X)=\left.\frac{d}{d t} g e^{t X} g^{-1}\right|_{t=0}$ denote the adjoint action of $G$ on its Lie algebra (see, e.g., Marsden and Ratiu [1994]). Thus $A d_{g}=$ $L_{g *} R_{g^{-1}}$ where $L_{g *}$ and $R_{g *}$ denote the left and right action of $G$ on its tangent bundle respectively; $L_{g}$ and $R_{g}$ are the left and right actions of the group on itself. Then we have:

Lemma 2.

$$
\begin{gathered}
D_{R} \phi_{g} \circ A d_{g^{-1}}=D_{L} \phi_{g} \\
A d_{g}\left(D_{R} \phi_{g}^{\#}\right)=\left(D_{L} \phi_{g}^{\#}\right) .
\end{gathered}
$$

Proof. We have

$$
\begin{aligned}
& D_{R} \phi_{g}(X)=\left.\frac{d}{d t}\right|_{t=0} \phi\left(L_{g} e^{t x}\right)=\phi_{*} L_{g *} X \\
& D_{L} \phi_{g}(X)=\left.\frac{d}{d t}\right|_{t=0} \phi\left(R_{g} e^{t x}\right)=\phi_{*} R_{g *} X .
\end{aligned}
$$

Hence $D_{R} \phi_{g} \circ A d_{g^{-1}}=D_{L} \phi_{g}$ proving 2.3. Thus

$$
\left\langle D_{R} \phi_{g}^{\#}, A d_{g^{-1}} X\right\rangle=\left\langle D_{L} \phi_{g}^{\#}, X\right\rangle
$$

or

$$
\left\langle A d_{g} D_{R} \phi_{g}^{\#}, X\right\rangle=\left\langle D_{L} \phi_{g}^{\#}, X\right\rangle
$$

by $A d$-invariance of the inner product. This gives (2.4).
Now in order to check the Jacobi identity in our Poisson bracket we also need the second derivatives of functions $\phi \in C^{\infty}(G)$. We make the following (natural) definitions:

Definition 3. Let $X, Y \in \mathfrak{g}$. We define

$$
\begin{aligned}
& D_{R}^{2} \phi_{g}(X, Y)=\left.\left.\frac{d}{d t}\right|_{t=0} \frac{d}{d s}\right|_{s=0} \phi\left(g e^{s Y} e^{t X}\right) \\
& D_{L}^{2} \phi_{g}(X, Y)=\left.\left.\frac{d}{d t}\right|_{t=0} \frac{d}{d s}\right|_{s=0} \phi\left(e^{t X} e^{s Y} g\right) \\
& D_{R} D_{L} \phi_{g}(X, Y)=\left.\left.\frac{d}{d t}\right|_{t=0} \frac{d}{d s}\right|_{s=0} \phi\left(e^{t X} g e^{s Y}\right)
\end{aligned}
$$

$$
D_{L} D_{R} \phi_{g}(X, Y)=\left.\left.\frac{d}{d t}\right|_{t=0} \frac{d}{d s}\right|_{s=0} \phi\left(e^{s Y} g e^{t X}\right) \text {. }
$$

Then we have

Lemma 4.

(i) $D_{R} D_{L} \phi_{g}(X, Y)=D_{L} D_{R} \phi_{g}(Y, X)$

(ii) $D_{R}^{2} \phi_{g}(X, Y)-D_{R}^{2} \phi_{g}(Y, X)=D_{R} \phi_{g}([X, Y])$

(iii) $D_{L}^{2} \phi_{g}(X, Y)-D_{L}^{2} \phi_{g}(Y, X)=D_{L} \phi_{g}([Y, X])$.

Proof. (i) follows directly from the definitions. To prove (ii) and (iii) note that $\left(D_{R} \phi_{g}\right)(X)=X_{g}^{L}(\phi)$ and $\left(D_{L} \phi_{g}\right)(X)=X_{g}^{R}(\phi)$ where $X_{g}^{L}=L_{g^{*}} X$ and $X_{g}^{R}=R_{g^{*}} X$ are the left- and right-invariant vector fields determined by $X \in \mathfrak{g}$, respectively. Thus, $D_{R}^{2} \phi_{g}(X, Y)=X_{g}^{L}\left(Y^{L}(\phi)\right)$ and, by a similar argument, $D_{R}^{2} \phi_{g}(Y, X)=Y_{g}^{L}\left(X^{L}(\phi)\right)$. This gives (ii) and (iii) follows in the same way.

\subsection{The Poisson Bracket Structure}

We now introduce (see Semenev-Tian-Shansky [1985], Deift and Li [1991]) an important class of Poisson structures on Lie groups via classical $r$ matrices, or, more specifically, via operators satisfying the Yang-Baxter identity (sometimes called the modified Yang-Baxter equation). Operators of this type yield a Poisson bracket usually known as the Sklyanin bracket.

Definition 5. A linear operator $A$ is said to satisfy the Yang-Baxter identity if $A: \mathfrak{g} \rightarrow \mathfrak{g}$ satisfies

(i) $[A X, A Y]-A([A X, Y]+[X, A Y])=[X, Y]$

(ii) $\langle A X, Y\rangle+\langle X, A Y\rangle=0$. 
An operator $A$ is called a classical r-matrix if

$$
[X, Y]_{A}=\frac{1}{2}([A X, Y]+[X, A Y])
$$

defines a Lie bracket on $\mathfrak{g} \cdot\left(\mathfrak{g},[\cdot, \cdot]_{A}\right)$ is called a Baxter Lie algebra.

Semenov-Tian-Shansky showed the following:

Lemma 6. An operator satisfying the Yang-Baxter identity is a classical $r$-matrix.

Lemma 7. If $A$ satisfies the Yang-Baxter identity, then

$\{\phi, \psi\}_{S}=\frac{1}{2}\left(A D_{R} \phi^{\#}, D_{R} \phi^{\#}\right)-\frac{1}{2}\left(A D_{L} \phi^{\#}, D_{L} \phi^{\#}\right)$

defines a Poisson structure on $G$ called the Sklyanin bracket.

Deift and Li [1991] used an extension of this bracket to analyze the Toda lattice equations. This extension employed operators satisfying the Yang-Baxter identity. Here we describe a generalization of the Sklyanin bracket making explicit the exact role of the Yang-Baxter identity in the proof of the Jacobi identity for the corresponding Poisson structure.

Definition 8. A linear mapping $J: \mathfrak{g} \rightarrow \mathfrak{g}$ is called an equivariant Yang-Baxter operator if, for some smooth function $F_{J}$,

$$
[J X, J Y]-J[J X, Y]-J[X, J Y]=F_{J}(X, Y),
$$

where

$$
A d_{g} F_{J}(X, Y)=F_{J}\left(A d_{g} X, A d_{g} Y\right)
$$

and if

$$
\langle J X, Y\rangle+\langle X, J Y\rangle \equiv 0 .
$$

We have the following result.

Theorem 9. Let $J_{1}$ and $J_{2}$ be equivariant YangBaxter operators with $F_{J_{1}}=F_{J_{2}} \equiv F_{J}$. Then

$$
\begin{aligned}
& \{\phi, \psi\}^{ \pm} \\
& =\frac{1}{2}\left(\left\langle D_{L} \phi^{\#}, J_{1} D_{L} \psi^{\#}\right\rangle \pm\left\langle D_{R} \phi^{\#}, J_{2}, D_{R} \psi^{\#}\right\rangle\right)
\end{aligned}
$$

is a Poisson bracket on $G$.

Proof. The bracket is clearly skew symmetric and bilinear. To prove the Jacobi identity, proceed as follows:

$$
\begin{aligned}
4\left\{\phi_{1},\left\{\phi_{2}, \phi_{3}\right\}^{ \pm}\right\}^{ \pm} \\
=2\left\langle D_{L} \phi_{1}^{\#}, J_{1}\left(D_{L}\left\{\phi_{2}, \phi_{3}\right\}^{ \pm}\right)\right\rangle \\
\quad \pm 2\left\langle D_{R} \phi_{1}^{\#}, J_{2}\left(D_{R}\left\{\phi_{2}, \phi_{3}\right\}^{ \pm}\right)^{\#}\right\rangle \\
=\left\langle D_{L} \phi_{1}^{\#}, J_{1}\left(D_{L}\left\langle D_{L} \phi_{2}^{\#}, J_{1} D_{L} \phi_{3}^{\#}\right\rangle\right)^{\#}\right\rangle \\
\quad \pm\left\langle D_{L} \phi_{1}^{\#}, J_{1}\left(D_{L}\left\langle D_{R} \phi_{2}^{\#}, J_{2} D_{R} \phi_{3}^{\#}\right\rangle\right)^{\#}\right\rangle \\
\quad \pm\left\langle D_{R} \phi_{1}^{\#}, J_{2}\left(D_{R}\left\langle D_{L} \phi_{2}^{\#}, J_{1}, D_{L} \phi_{3}^{\#}\right\rangle\right)^{\#}\right\rangle \\
\quad+\left\langle D_{R} \phi_{1}^{\#}, J_{2}\left(D_{R}\left\langle D_{R} \phi_{2}^{\#}, J_{2} D_{R} \phi_{3}^{\#}\right\rangle\right)^{\#}\right\rangle .
\end{aligned}
$$

Since $J_{1}$ and $J_{2}$ are skew and $\left\langle D_{L(R)} \phi^{\#}, X\right\rangle=$ $D_{L(R)} \phi(X)$ by definition, we may rewrite this expression as

$$
\begin{aligned}
4\left\{\phi_{1},\left\{\phi_{2}, \phi_{3}\right\}^{ \pm}\right\}^{ \pm} & =-D_{L}^{2} \phi_{2}\left(J_{1} D_{L} \phi_{3}^{\#}, J_{1} D_{L} \phi_{1}^{\#}\right) \\
& +D_{L}^{2} \phi_{3}\left(J_{1} D_{2} \phi_{2}^{\#}, J_{1} D_{L} \phi_{1}^{\#}\right) \\
& -D_{R}^{2} \phi_{2}\left(J_{2} D_{R} \phi_{3}^{\#}, J_{2} D_{R} \phi_{1}^{\#}\right) \\
& +D_{R}^{2} \phi_{3}\left(J_{2} D_{R} \phi_{2}^{\#}, J_{2} D_{R} \phi_{1}^{\#}\right) \\
& \mp D_{L} D_{R} \phi_{2}\left(J_{2} D_{R} \phi_{3}^{\#}, J_{1} D_{L} \phi_{1}^{\#}\right) \\
& \pm D_{L} D_{R} \phi_{3}\left(J_{2} D_{R} \phi_{2}^{\#}, A_{1} D_{2} \phi_{1}^{\#}\right) \\
& \mp D_{R} D_{L} \phi_{2}\left(J_{1} D_{L} \phi_{3}^{\#}, J_{2} D_{R} \phi_{1}^{\#}\right) \\
& \pm D_{R} D_{L} \phi_{3}\left(J_{1} D_{L} \phi_{2}^{\#}, J_{2} D_{R} \phi_{1}^{\#}\right) .
\end{aligned}
$$

We need to show that this expression plus its cyclic permutations is identically zero. To do this, represent the first two terms in (2.17) in obvious fashion as $-(2,3,1)+(3,2,1)$. This plus its permutations is the expression

$$
\begin{aligned}
& -(2,3,1)+(3,2,1) \\
& -(3,1,2)+(1,3,2) \\
& -(1,2,3)+(2,1,3) .
\end{aligned}
$$

Using Lemma 4 (iii) this equals

$$
\begin{aligned}
& \left\langle D_{L} \phi_{2}^{\#},\left[J_{1} D_{L} \phi_{3}^{\#}, J_{1} D_{L} \phi_{1}^{\#}\right]\right\rangle \\
& \quad+\left\langle D_{L} \phi_{1}^{\#},\left[J_{1} D_{L} \phi_{2}^{\#}, J_{1} D_{L} \phi_{3}^{\#}\right]\right\rangle \\
& \quad+\left\langle D_{L} \phi_{3}^{\#},\left[J_{1} D_{L} \phi_{1}^{\#}, J_{1} D_{L} \phi_{2}^{\#}\right]\right\rangle .
\end{aligned}
$$

Now use the facts that $J_{1}$ is skew and $\langle[X, Y], Z\rangle+$ $\langle Y,[X, Z]\rangle=0$ to rewrite this as

$$
\begin{aligned}
& \left\langle D_{L} \phi_{1}^{\#},\left[J_{1} D_{L} \phi_{2}^{\#}, J_{1} D_{L} \phi_{3}^{\#}\right]\right\rangle \\
& -\left\langle D_{L} \phi_{1}^{\#}, J_{1}\left[D_{L} \phi_{2}^{\#}, J_{1} D_{L} \phi_{3}^{\#}\right]\right\rangle \\
& -\left\langle D_{L} \phi^{\#}, J_{1}\left[J_{1} D_{L} \phi_{2}^{\#}, D_{L} \phi_{3}^{\#}\right]\right\rangle .
\end{aligned}
$$

Since $J_{1}$ is an equivariant Yang-Baxter operator this equals

$$
-\left\langle D_{L} \phi_{1}^{\#}, F_{J}\left(D_{L} \phi_{2}^{\#}, D_{L} \phi_{3}^{\#}\right)\right\rangle .
$$

Similarly, now using Lemma 4 (ii), we obtain for the second two terms of (2.17),

$$
+\left\langle D_{R} \phi_{1}^{\#}, F_{J}\left(D_{R} \phi_{2}^{\#}, D_{R} \phi_{3}^{\#}\right)\right\rangle .
$$

By Lemma 2, we have $\left(D_{L} \phi_{g}\right)^{\#}=A d_{g}\left(D_{R} \phi_{g}^{\#}\right)$. Thus, since $\langle$,$\rangle is A d$-invariant and $F_{J}$ is $A d$ equivariant, the cyclic sum of terms 1 and 2 in (2.17) is identically zero. 
Now consider the last four terms of (2.17). By (i) of Lemma 4 we may rewrite these terms as

$$
\begin{aligned}
& \mp D_{L} D_{R} \phi_{2}\left(J_{2} D_{R} \phi_{3}^{\#}, J_{1} D_{L} \phi_{1}^{\#}\right) \\
& \pm D_{L} D_{R} \phi_{3}\left(J_{2} D_{R} \phi_{2}^{\#}, J_{1} D_{L} \phi_{1}^{\#}\right) \\
& \mp D_{L} D_{R} \phi_{2}\left(J_{2} D_{R} \phi_{1}^{\#}, J_{1} D_{L} \phi_{3}^{\#}\right) \\
& \pm D_{L} D_{R} \phi_{3}\left(J_{2} D_{R} \phi_{1}^{\#}, J_{1} D_{L} \phi_{2}^{\#}\right) .
\end{aligned}
$$

Again, represent this as

$$
\mp(2,3,1) \pm(3,2,1) \mp(2,1,3) \pm(3,1,2) .
$$

Then the cyclic sum is

$$
\begin{aligned}
& \mp(2,3,1) \pm(3,2,1) \mp(2,1,3) \pm(3,1,2) \\
& \mp(3,1,2) \pm(1,3,2) \mp(3,2,1) \pm(1,2,3) \\
& \mp(1,2,3) \pm(2,1,3) \mp(1,3,2) \pm(2,3,1)
\end{aligned}
$$

which is identically zero. Hence the Jacobi identity is satisfied.

The proof of lemma 7 follows directly from this result by noting that $F_{J}(X, Y)=[X, Y]$, is clearly $A d$-invariant.

\section{DYNAMICS WITH RESPECT TO AN ALMOST POISSON STRUCTURE}

In this section we consider dynamics on groups under the bracket $(2.16)$, but in the case where $F_{J}$ is generally not $A d_{g}$ equivariant - such structures will be called almost Poisson structures on Lie groups. We begin by considering the general case and then specialize to the case of particular interest to us - cross products of Lie groups.

Such a bracket is an instance of an almost Poisson structure (see da Silva and Weinstein [1999]):

Definition 10. An almost Poisson manifold is a pair $(M,\{\}$,$) where M$ is a smooth manifold and (i) $\{$,$\} defines an almost Lie algebra structure$ on the $C^{\infty}$ functions on $M$, i.e. the bracket satisfies all conditions for a Lie algebra except that the Jacobi identity is not satisfied and (ii) $\{$,$\} is$ a derivation in each factor.

Given an almost Poisson structure with local coordinate expression $\pi^{i j}(z)$ (the generalization of the Poisson tensor) and a function $H$ on $M$, one defines an almost Poisson vector field on $M$ by

$$
\dot{z}^{i}=\pi^{i j}(z) \frac{\partial H}{\partial z^{j}} .
$$

Now in our setting we have:

Theorem 11. The equation

$$
\dot{\phi}=\{\phi, H\}^{ \pm}
$$

where $\{,\}^{ \pm}$is the bracket (2.16) on a Lie group $G$ yields the equation

$$
\dot{g}=\frac{1}{2} R_{g *} J_{1} D_{L} H^{\#} \pm \frac{1}{2} L g_{*} J_{2} D_{R} H^{\#} .
$$

If $H \in I(G)$, the functions on $G$ invariant under conjugation, then $D_{L} H=D_{R} H=D H$ and (3.2) becomes

$$
\dot{g}=\frac{1}{2} R_{g^{*}} J_{1} D H^{\#} \pm \frac{1}{2} L_{g^{*}} J_{2} D H^{\#}
$$

Proof. Equation (3.2) may be written as

$$
\frac{d}{d t} \phi(g)=\frac{1}{2} D_{L} \phi\left(J_{1} D_{L} H^{\#}\right) \pm \frac{1}{2} D_{R} \phi\left(J_{2} D_{R} H^{\#}\right) .
$$

But

$D_{L} \phi=\phi_{*} R_{g^{*}}, D_{R} \phi=\phi_{*} L_{g^{*}}$ and $\frac{d}{d t} \phi(g)=\phi_{*} \dot{g}$.

Hence we obtain (3.3).

Now, if $\phi \in I(G)$, i.e., $\phi(g)=\phi\left(h g h^{-1}\right)$, for all $h \in G$, then $\phi_{*} X=\phi_{*} A d_{h} X$, for all $h \in G, X \in \mathfrak{g}$. Hence $\phi_{*}=\phi_{*} A d_{h}$ or $\phi_{*} R_{h_{*}}=\phi_{*} L_{h_{*}}$ and thus

$$
D_{L} \phi_{g}=\phi_{*} R_{g_{*}}=\phi_{*} L_{g_{*}}=D_{R} \phi_{g} \text {. }
$$

We now consider the case of interest to us, where the Lie group is

$$
D=G \times G
$$

with Lie algebra $\mathfrak{g} \times \mathfrak{g} \equiv \mathfrak{g}^{D}$. We define the map $J: \mathfrak{g}^{D} \rightarrow \mathfrak{g}^{D}$ given by

$$
J\left(X_{1}, X_{2}\right)=\left(X_{2},-X_{1}\right)
$$

for $\left(X_{1}, X_{2}\right) \in \mathfrak{g}^{D}$.

We shall shortly define a bracket using $J$ but note that $J$ does not define a Poisson structure, for while $J$ is clearly skew, if $X, Y \in \mathfrak{g}^{D}$

$$
\begin{aligned}
F_{J}(X, Y)= & {[J X, J Y]-J[J X, Y]-J[X, J Y] } \\
= & \left(\begin{array}{l}
{\left[X_{1}+X_{2}, Y_{1}+Y_{2}\right]} \\
{\left[X_{1}+X_{2}, Y_{1}+Y_{2}\right]}
\end{array}\right) \\
& -\left(\begin{array}{l}
{\left[X_{1}, Y_{1}\right]} \\
{\left[X_{2}, Y_{2}\right]}
\end{array}\right)
\end{aligned}
$$

which is clearly not $A d_{g}$ invariant.

We now make the following definitions in this setting in order to set up the equations of motion:

Definition 12. Let $e$ be the identity in $G, g \in D$, $\left(X_{1}, X_{2}\right) \in \mathfrak{g}^{D}$, then we define

$$
\begin{aligned}
& \left(D_{R}^{1} \phi\right)_{g}\left(X_{1}\right)=\left.\frac{d}{d t}\right|_{t=0} \phi\left(g\left(e^{t X_{1}}, e\right)\right) \\
& \left(D_{R}^{2} \phi\right)_{g}\left(X_{2}\right)=\left.\frac{d}{d t}\right|_{t=0} \phi\left(g\left(e, e^{t X_{2}}\right)\right) \\
& \left(D_{L}^{1} \phi\right)_{g}\left(X_{1}\right)=\left.\frac{d}{d t}\right|_{t=0} \phi\left(\left(e^{t X_{1}}, e\right) g\right) \\
& \left(D_{L}^{2} \phi\right)_{g}\left(X_{2}\right)=\left.\frac{d}{d t}\right|_{t=0} ^{\phi} \phi\left(\left(e, e^{t X_{2}}\right) g\right) \\
& \left(D_{R} \phi\right)_{g}\left(X_{1}, X_{2}\right)=\left(\left(D_{R}^{1} \phi\right)_{g},\left(D_{R}^{2} \phi\right)_{g}\right)\left(X_{1}, X_{2}\right) \\
& \left(D_{L} \phi\right)_{g}\left(X_{1}, X_{2}\right)=\left(\left(D_{L}^{1} \phi\right)_{g},\left(D_{L}^{2} \phi\right)_{g}\right)\left(X_{1}, X_{2}\right) .
\end{aligned}
$$

Using the inner product on $\mathfrak{g}$ and $\mathfrak{g}^{D}$ we get obvious definitions of $\left(D_{L}^{1} \phi_{g}\right)^{\#} \in \mathfrak{g}$, etc. Then the + bracket (2.16) becomes 


$$
\begin{aligned}
\{\phi, \psi\}= & \frac{1}{2}\left\langle D_{L}^{1} \phi^{\#}, D_{L}^{2} \psi^{\#}\right\rangle-\frac{1}{2}\left\langle D_{L}^{2} \phi^{\#}, D_{L}^{1} \psi^{\#}\right\rangle \\
& +\frac{1}{2}\left\langle D_{R}^{1} \phi^{\#}, D_{R}^{2} \psi^{\#}\right\rangle \\
& -\frac{1}{2}\left\langle D_{R}^{2} \phi^{\#}, D_{R}^{1} \psi^{\#}\right\rangle
\end{aligned}
$$

Using (3.3), we have

Corollary 13. The equations $\dot{\phi}=\{\phi, H\}$ induced by the bracket (3.7) on $D$ are given by

$\dot{g}_{1}=\frac{1}{2} R_{g_{1}^{*}}\left(D_{L}^{2} H_{\left(g_{1}, g_{2}\right)}\right)^{\#}+\frac{1}{2} L_{g_{1}^{*}}\left(D_{R}^{2} H_{\left(g_{1}, g_{2}\right)}\right)^{\#}$

$\dot{g}_{2}=-\frac{1}{2} R_{g_{2}^{*}}\left(D_{L}^{1} H_{\left(g_{1}, g_{2}\right)}\right)^{\#}-\frac{1}{2} L_{g_{2}^{*}}\left(D_{R}^{1} H_{\left(g_{1}, g_{2}\right)}\right)$.

We now prove the main result of the paper, which provides a special case of these equations and turns out to be intimately related to the generalized rigid body equations on $s o(n)$.

Theorem 14. Consider the flow (3.8) in the case

$$
H=4 \operatorname{Trace}\left(I_{1} g_{2}^{-1} g_{1}+I_{2} g_{1} g_{2}^{-1}\right)
$$

where $I_{1}$ and $I_{2}$ are symmetric, positive definite matrices. Let $J_{1}$ and $J_{2}$ be defined by

$$
\begin{aligned}
& J_{1}^{-1}(X)=I_{1} X+X I_{1} \\
& J_{2}^{-1}(X)=I_{2} X+X I_{2} .
\end{aligned}
$$

Then the flow (3.8) is given by

$$
\begin{aligned}
\dot{g}_{1} & =J_{2}^{-1}\left(g_{2} g_{1}^{-1}-g_{1} g_{2}^{-1}\right) g_{1}-g_{1} J_{1}^{-1}\left(g_{2}^{-1} g_{1}-g_{1}^{-1} g_{2}\right) \\
\dot{g}_{2} & =J_{2}^{-1}\left(g_{2} g_{1}^{-1}-g_{1} g_{2}^{-1}\right) g_{2} \\
& -g_{2} J_{1}^{-1}\left(g_{2}^{-1} g_{1}-g_{1}^{-1} g_{2}\right) .
\end{aligned}
$$

Proof. Let $g=\left(g_{1}, g_{2}\right)$ and compute as follows:

$D_{R}^{2} H_{g}(X)=4 \operatorname{Trace}\left(-I_{1} X g_{2}^{-1} g_{1}-I_{2} g_{1} X g_{2}^{-1}\right)$

$=4 \operatorname{Trace}\left(-J_{1}^{-1}(X) g_{2}^{-1} g_{1}+X I_{1}, g_{2}^{-1} g_{1}-g_{2}^{-1} I_{2} g_{1} X\right)$

$=4 \operatorname{Trace}\left(-X J_{1}^{-1}\left(g_{2}^{-1} g_{1}\right)+X I_{1}, g_{2}^{-1} g_{1}-g_{2}^{-1} I_{2} g_{1} X\right)$

$=2 \operatorname{Trace}\left(-X J_{1}^{-1}\left(g_{2}^{-1} g_{1}-g_{1}^{-1} g_{2}\right)\right.$

$\left.+X\left(I_{1} g_{2}^{-1} g_{1}-g_{1}^{-1} g_{2} I_{1}\right)+\left(g_{1}^{-1} I_{2} g_{2}-g_{2}^{-1} I_{2} g_{1}\right) X\right)$

since $X \in s o(n)$ and $g_{1} g_{2} \in S O(n)$. Therefore,

$$
\begin{aligned}
& \left(D_{R}^{2} H_{g}\right)^{\#}=-2 J_{1}^{-1}\left(g_{2}^{-1} g_{1}-g_{1}^{-1} g_{2}\right) \\
& +2\left(I_{1} g_{2}^{-1} g_{1}-g_{1}^{-1} g_{2} I_{1}\right)+2\left(g_{1}^{-1} I_{2} g_{2}-g_{2}^{-1} I_{2} g_{1}\right) .
\end{aligned}
$$

Computing similarly we find:

$$
\begin{aligned}
& \left(D_{L}^{2} H_{g}\right)^{\#}=-2 J_{2}^{-1}\left(g_{1} g_{2}^{-1}-g_{2} g_{1}^{-1}\right) \\
& +2\left(g_{1} g_{2}^{-1} I_{2}-I_{2} g_{2} g_{1}^{-1}\right)+2\left(g_{2} I_{1} g_{1}^{-1}-g_{1} I_{1} g_{2}^{-1}\right) \\
& \left(D_{L}^{1} H_{g}\right)^{\#}=2 J_{2}^{-1}\left(g_{1} g_{2}^{-1}-g_{2} g_{1}^{-1}\right) \\
& +2\left(g_{2} g_{1}^{-1} I_{2}-I_{2} g_{1} g_{2}^{-1}\right)+2\left(g_{1} I g_{2}^{-1}-g_{2} I_{1} g_{1}^{-1}\right) \\
& \left(D_{R}^{1} H_{g}\right)^{\#}=2 J_{1}^{-1}\left(g_{2}^{-1} g_{1}-g_{1}^{-1} g_{2}\right) \\
& +2\left(I_{1} g_{1}^{-1} g_{2}-g_{2}^{-1} g_{1} I_{1}\right)+2\left(g_{2}^{-1} I_{2} g_{1}-g_{1}^{-1} I_{2} g_{2}\right) .
\end{aligned}
$$

Substitution into (3.8) gives the result.

\section{THE RIGID BODY EQUATIONS}

In this last section we relate the system (3.10) to the rigid body system on $S O(n)$. We show

Theorem 15. The equations (3.10) in the case $J_{1}^{-1}=0, J_{2}=J$ are locally equivalent to the generalized rigid body equations.

To do this, we recall some background information from Bloch, Brockett, and Crouch [1997] (see also Bloch, Crouch, Marsden, and Ratiu [1998, 2000]). We recall that the rigid body equations on $S O(n)$ (or generally on any compact Lie group - see e.g. Marsden and Ratiu [1994], Ratiu [1980]) may be written as

$$
\begin{aligned}
\dot{Q} & =\Omega Q \\
\dot{M} & =[\Omega, M]
\end{aligned}
$$

where $Q \in S O(n)$ denotes the configuration space variables, $\Omega \in \operatorname{so}(n)$ is the angular velocity, and $M=J \Omega=\Lambda \Omega+\Omega \Lambda$ is the angular momentum. Here $J$ is a symmetric positive definite operator defined by the diagonal positive definite matrix $\Lambda$. We remark that the rigid body equations here are written in right-invariant as opposed to the commonly used left-invariant form in order to be consistent with the conventions used in the remainder of the paper. This results in a sign change in the second of equations (4.1). The classical rigid body equations (4.1) are of course Hamiltonian on $T^{*} S O(n)$ with respect to the canonical symplectic structure. We now consider the following equations:

$$
\begin{aligned}
& \dot{Q}=\Omega Q \\
& \dot{P}=\Omega P,
\end{aligned}
$$

where $\Omega=J^{-1} M$ and $M=P Q^{T}-Q P^{T}$ for $Q, P \in S O(n)$. We then can easily check that:

Proposition 16. The mapping $(Q, P) \mapsto(Q, M)$, from $S O(n) \times S O(n)$ to $T^{*} S O(n)$ takes all solutions of equation (4.2) onto solutions of the generalized rigid body equations (4.1).

Proof. Differentiating $M=P Q^{T}-Q P^{T}$ and using the equations (4.2) gives the second of equations (4.1).

Conversely, given the rigid body equations (4.1) we may solve for the variable $P$ in the expression

$$
M=P Q^{T}-Q P^{T}
$$

in a neighborhood of $M=0$. Locally, in a neighborhood of $M=0$, where $\sinh ^{-1}$ is well defined

$$
P=\left(e^{\sinh ^{-1} M / 2}\right) Q .
$$


This follows from the observation that

$$
M=e^{\sinh ^{-1} M / 2}-e^{-\sinh ^{-1} M / 2} .
$$

For $s o(n)$, however, sinh is many to one, so the two representations are not entirely equivalent. (For more details giving the precise region for $M$ for which one can solve for $P$, see Bloch, Crouch, Marsden, and Ratiu [2000]. This reference also contains a discussion of how this system is related to certain optimal control problems.)

Observe, however, that this proves Theorem 15, as this new form of the rigid body equation is exactly of the type given to us by our almost Poisson structure, and the system of equations (3.10), under the conditions of theorem 15

We would like to say some more, however, about the Hamiltonian structure of these equations, again following Bloch, Brockett, and Crouch [1997].

Note firstly that the generalized rigid body flow naturally reduces to a flow in the variable $M$ on an adjoint orbit of $s o(n)$ and we can view the map which takes $P Q^{T}-Q P^{T}$ to $M$ as reduction. In fact, the map $(Q, P) \mapsto(Q, M)$ given above is a canonical transformation from the symplectic structure on $T^{*} g l(n)$ to that on $T^{*} S O(n)$ which intertwines the Hamiltonian equations (4.2) on $T^{*} g l(n)$ with the Hamiltonian equations (4.1) on $T^{*} S O(n)$.

While the classical rigid body equations (4.1) are Hamiltonian on $T^{*} S O(n)$ with respect to the canonical symplectic structure, on the group we have

Proposition 17. The generalized rigid body equations in the form (4.2) are Hamiltonian on $T^{*} g l(n)$ with respect to the canonical symplectic structure and the Hamiltonian

$$
H=\frac{1}{4}\left\langle J^{-1}\left(P Q^{T}-Q P^{T}\right), P Q^{T}-Q P^{T}\right\rangle,
$$

where $\langle\xi, \eta\rangle=\operatorname{Trace}\left(\xi^{T} \eta\right)$.

This is a straightforward computation.

We remark that here $P$ and $Q$ are natural coordinates for $T^{*} g l(n)$ and, for $P(0), Q(0) \in S O(n)$, $P(t)$ and $Q(t)$ evolve in $S O(n)$ under the flow of $H$. Hence $S O(n) \times S O(n)$ is an invariant manifold for the flow of $H$. Note also that this Hamiltonian is equivalent to $H=(1 / 4)\left\langle J^{-1} M, M\right\rangle$, as in Ratiu [1980].

\section{REFERENCES}

Bloch, A.M., R.W. Brockett, and T.S. Ratiu [1992] Completely integrable gradient flows. Comm. Math. Phys. 147, 57-74.

Bloch, A.M., R. W. Brockett, and P.E. Crouch [1997] Completely integrable gradient flows. Comm. Math. Phys. 187, 357-373.

Bloch, A.M. and P.E. Crouch [1995] On the geometry of optimal control and geodesic flows Proc. CDC 34, IEEE, 3283-3288.

Bloch, A. M. and P. E. Crouch [1996] Optimal control and geodesic flows Systems and Control Letters 28, 65-72.

Bloch, A.M., P.E. Crouch, J.E. Marsden, and T.S. Ratiu [1998] Discrete rigid body dynamics and optimal control Proc. $C D C$ 37, IEEE, 2249-2254.

Bloch, A.M., P.E. Crouch, J.E. Marsden and T.S. Ratiu [2000] Optimal control and discrete rigid body equations, to appear.

Bloch, A.M., H. Flaschka, and T.S. Ratiu [1990] A convexity theorem for isospectral sets of Jacobi matrices in a compact Lie algebra. Duke Math. J. 61, 41-66.

Brockett, R.W. [1994] The double bracket equation as a solution of a variational problem. Fields Institute Comm. 3, 69-76.

Cannas da Silva, A. and A. Weinstein [1999] Geometric Models for Noncommutative Algebras, American Mathematical Society.

P. Deift and L.-C, Li [1991] Poisson geometry of the Analog of the Miura maps and BacklundDarboux Transformations for equations of Toda type and periodic Toda flows, Communications in Mathematical Physics 143, 201-214.

Drinfeld, V. [1983], Hamiltonian structures on Lie groups, Lie bialgebras and the geometric meaning of the classical Yang-Baxter equations, Sov. Math. Dokl. 27, 68-71.

Lu, J. and A. Weinstein [1990] Poisson Lie groups, dressing transformations, and the Bruhat decomposition J. Diff. Geom 31, 501-526.

Marsden, J.E. and T.S. Ratiu [1994] Introduction to Mechanics and Symmetry. Texts in Applied Mathematics, 17, Springer-Verlag, 1994. Second Edition, 1999.

Ratiu, T. [1980] The motion of the free ndimensional rigid body. Indiana U. Math. J., 29, 609-627.

Semenov-Tain Shansky [1985], Dressing transformations and Poisson group actions Publ. RIMS, Kyoto University, 21, 1237-1260.

Weinstein, A [1983], Local structure of Poisson manifolds, J. Diff. Geometry, 18, 523-558. 